\title{
Data rates of SubTHz wireless telecommunication channels
}

\author{
G.M. Bubnov, I.V. Lesnov and V.F. Vdovin
}

Institute of Applied Physics RAS, Nizhniy Novgorod, Russia, vdovin_iap@mail.ru

The work is devoted to the actual problem of data rate of wireless telecommunication channels. Presented analysis of the telecommunication channel of subterahertz (subTHz) frequency range - as the most promising band for the implementation of wireless telecommunications for space links and terrestrial communications of high capacity.

There are some market researches, indicating a big and increasing interest to $\mathrm{THz}$ communications [1].

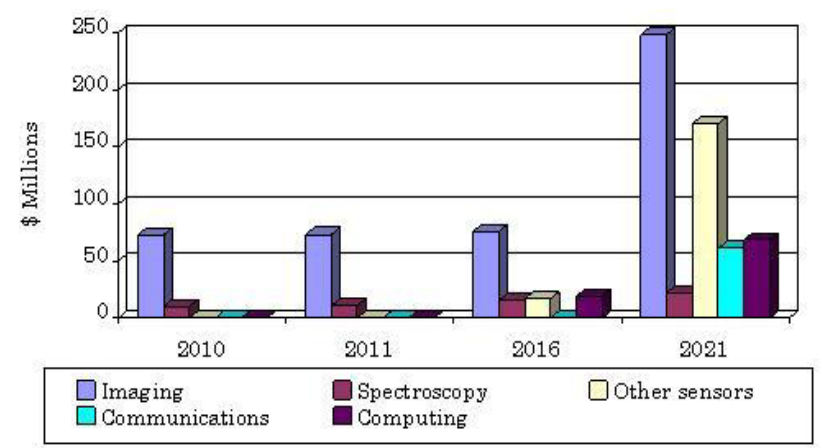

Fig. 1. Forecast of the THz telecommunication market potential [1]

Channel is considered as a combination of technical realization of high effective transponder - transmitter together with the atmospheric open line. The means to achieve a high signal/noise ratio and high data rates is usage of low-noise cryogenic (subTHz) receivers actively developing in IAP for radioastronomy and atmosphere spectroscopy [2]. The theoretical analysis of data rates for various natural and technical implementa-tions of communication channels demonstrated reasonable limits of cooling of receivers about $\sim 10 \mathrm{~K}$, providing a weighty increase channel capacity, while deeper cooling is impractical due to atmosphere restrictions in certain ranges and conditions of signal propagation, including altitude and seasonal features. The presented theoretical analysis of data rates have been confirmed by experiments related to the implementation of the telecommunication channel Earth - Space for communication with deep space missions, performed in the framework of the international project Exo-Mars, launched on March 13, 2016. There are cooled down $7 \mathrm{~K}$ front end of receivers with total noise less then $20 \mathrm{~K}$ (including $6 \mathrm{~K}$ noise temperature of receivers) [3]. See Fig. 2.

The calculation of the capacity of wireless telecommunications was carried out on the basis of the the Shannon - Hartley theorem, under the Dryagin - Goronina limitations [4]:

$$
S N R=\frac{P_{\text {signal }}}{P_{\text {noise }}},
$$

where $\mathrm{P}_{\text {noise }}-$ is the power of additive white Gaussian noise and $\mathrm{P}_{\text {signal, }}$, power of signal.

The receiver noise temperature, or $T_{n}$, in the the Shannon - Hartley theorem would be a more accurate parameter then noise factor $(F)$. This value does not include the temperature $T_{o}$ which for cooled receivers (to get extremely high sensitivity, receivers normally are incorporated into the cryogenic cooling system) may significantly differ from the standard $T_{o}=300 \mathrm{~K}$. $T_{n}$ is related to $F$ through the well-known formula:

$$
\mathrm{F}=1+\mathrm{T}_{\mathrm{n}} / \mathrm{T}_{\mathrm{o}}
$$

A more precise formula for determining the signal-tonoise ratio of the receiver will therefore be as follows:

$$
\mathrm{SNR}=\mathrm{P}_{\mathrm{rx}} / \mathrm{kB}\left(\mathrm{T}_{\mathrm{n}}+\mathrm{T}_{\mathrm{o}}\right)
$$

thus yielding the developed Shannon formula for the link capacity estimation:

$$
\mathrm{C}=\mathrm{B} \log 2\left(1+\mathrm{P}_{\mathrm{rx}} / \mathrm{kB}\left(\mathrm{T}_{\mathrm{n}}+\mathrm{T}_{\mathrm{o}}\right)\right) .
$$

SNR of a total channel with atmosphere is determined by two models - free-path-lose attenuation and integral atmospheric attenuation:

$$
P_{\text {signal } d b}=P_{t x}+G_{t x}+G_{r x}+20 \log \frac{c}{4 \pi d v}-\alpha_{h},
$$

where $\alpha_{h}-$ integral total atmospheric attenuation from $h$ to $\sim 200 \mathrm{~km}$.

In our work we have analyzed capacity of channel Earth - Space depends of Equivalent receiver noise temperature, ambient temperature, altitude of a receiver location, angle of inclination relative to the zenith.

The best results are obviously for open space communications, however there are definite possibilities not

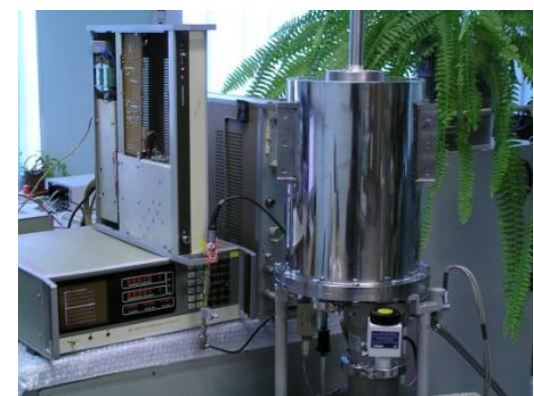

Cryo LNA in lab

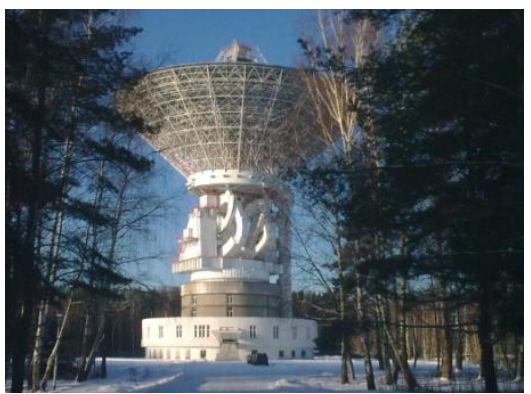

TNA 1500 antenna

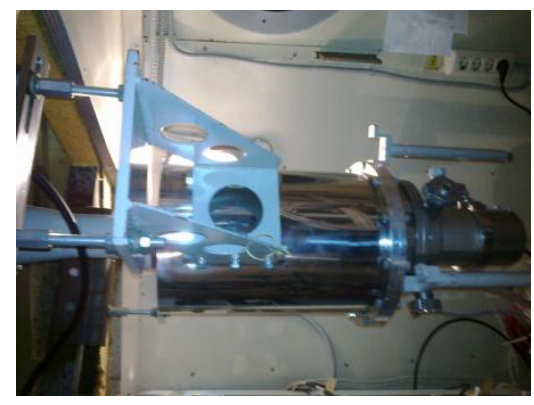

CryoLNA in focus TNA 1500

Fig. 2 
only for Space - Earth cannels but for terrestrial too, but only for atmospheric windows 1.3 and $0.8 \mathrm{~mm}$.

\section{References}

1. Gert de Lange. Valorization Seminar. TeraDec Meeting, Apr. 2012, SRON, Utrecht, the Netherlands.

2. V. F. Vdovin . and I. I. Zinchenko Modern Millimeter and Submillimeter receiver systems for Radio Astronomy. Radiophysics and Quantum Electronics, Vol. 52, No. 7, 2009, p. 461.
3. Cryogenically Cooled Low-Noise Amplifier for Radio-Astronomical Observations and Centimeter-Wave DeepSpace Communications Systems / V. F. Vdovin, V. G. Grachev, S. Yu. Dryagin, A. I. Eliseev, R. K. Kamaletdinov,D. V. Korotaev, I. V. Lesnov, M. A. Mansfeld, E. L. Pevzner, V. G. Perminov, A. M. Pilipenko, B. D. Sapozhnikov, V. P. Saurin // Astrophysical Bulletin - 2016. - V. 71. - № 1. - pp. 125128.

4. Yu.A. Dryagin and K.A. Goronina On the Noise Parameters of Diode Mixers. Journal of Radio Electronics, № 1, January 2002. 